\title{
Application of Photoacoustic Imaging for Lymphedema Treatment
}

\author{
Yushi Suzuki, MD, $\mathrm{PhD}^{1}$ \\ Hisashi Sakuma, MD² Nobuaki Imanishi, MD, PhD ${ }^{3}$ \\ ${ }^{1}$ Department of Plastic and Reconstructive Surgery, Keio University \\ School of Medicine, Tokyo, Japan \\ ${ }^{2}$ Department of Plastic and Reconstructive Surgery, Tokyo Dental \\ College Ichikawa General Hospital, Chiba, Japan \\ ${ }^{3}$ Department of Anatomy, Keio University School of Medicine, Tokyo, Japan \\ ${ }^{4}$ Luxonus Inc. Kawasaki, Japan
}

J Reconstr Microsurg 2022;38:254-262.

Watanabe, $\mathrm{MD}^{1} \quad$ Keisuke Okabe, MD PhD ${ }^{1}$ Sadakazu Aiso, MD, $\mathrm{PhD}^{3,4}$ Kazuo Kishi, MD, $\mathrm{PhD}^{1}$

Address for correspondence Yushi Suzuki, MD, PhD, Department of Plastic and Reconstructive Surgery, Keio University School of Medicine, 35 Shinanomachi, Shinjuku-ku, Tokyo 160-8582, Japan (e-mail: fi080150@keio.jp).

\begin{abstract}
Background Lymphatic vessels are difficult to identify using existing modalities as because of their small diameter and the transparency of the lymph fluid flowing through them.

Methods Here, we introduce photoacoustic lymphangiography (PAL), a new modality widely used for lymphedema treatment, to observe limb lymphatic vessels. The photoacoustic imaging system used in this study can simultaneously visualize lymphatic vessels and veins with a high resolution $(0.2 \mathrm{~mm})$ and can also observe their three-dimensional relationship with each other.

Results High-resolution images of the lymphatic vessels, detailed structure of the dermal back flow, and the three-dimensional positional relationship between the lymphatic vessels and veins were observed by PAL.

Conclusion The clear image provided by PAL could have a major application in pre- and postoperative use during lymphaticovenular anastomosis for lymphedema treatment.
\end{abstract}

Lymphatics are vessels that circulate throughout the body, similar to blood vessels. However, they are difficult to observe from an anatomical point of view and diagnose by radiology because of their small diameter and the transparency of the lymph fluid flowing through them.

Lymphoscintigraphy and near-infrared fluorescence (NIRF) lymphangiography have been used to observe lymphatic vessels for the diagnosis of lymphedema, which is caused by the congestion of lymphatic flow. However, the image resolution from these modalities is insufficient, and it is often difficult to clearly identify individual lymphatic vessel. Moreover, the information obtained from these modalities is two-dimensional, making it impossible to evaluate the depth or the three-dimensional relationships between lymph vessels and blood vessels. In this study, we imple- mented a photoacoustic imaging (PAI) system that uses the photoacoustic effect, which is a new imaging technique that can clearly visualize the vascular system, hence also visualizing the lymphatic vessels in detail and in three dimensions.

The principle of the PAI system can be summarized, as follows (-Fig. 1):

(1) A pulse near-infrared laser repeatedly irradiates the object to be photographed.

(2) The object absorbs light energy and converts it into thermal energy, causing a slight increase in temperature.

(3) When the temperature rises, the object undergoes a slight thermal expansion, resulting in a volume change.

(4) When a volume change occurs, a feeble ultrasound, called a photoacoustic wave, is generated. received

June 18, 2021

accepted after revision

September 13, 2021

published online

December 27, 2021
Issue Theme Ultrasound Use in Reconstructive Surgery; Guest Editor: Joon Pio Hong, MD, PhD, MMM

DOI https://doi.org/ $10.1055 / \mathrm{s}-0041-1736518$. ISSN 0743-684X.

\footnotetext{
(C) 2021. The Author(s).

This is an open access article published by Thieme under the terms of the Creative Commons Attribution-NonDerivative-NonCommercial-License, permitting copying and reproduction so long as the original work is given appropriate credit. Contents may not be used for commercial purposes, or adapted, remixed, transformed or built upon. (https://creativecommons.org/ licenses/by-nc-nd/4.0/)

Thieme Medical Publishers, Inc., 333 Seventh Avenue, 18th Floor, New York, NY 10001, USA
} 

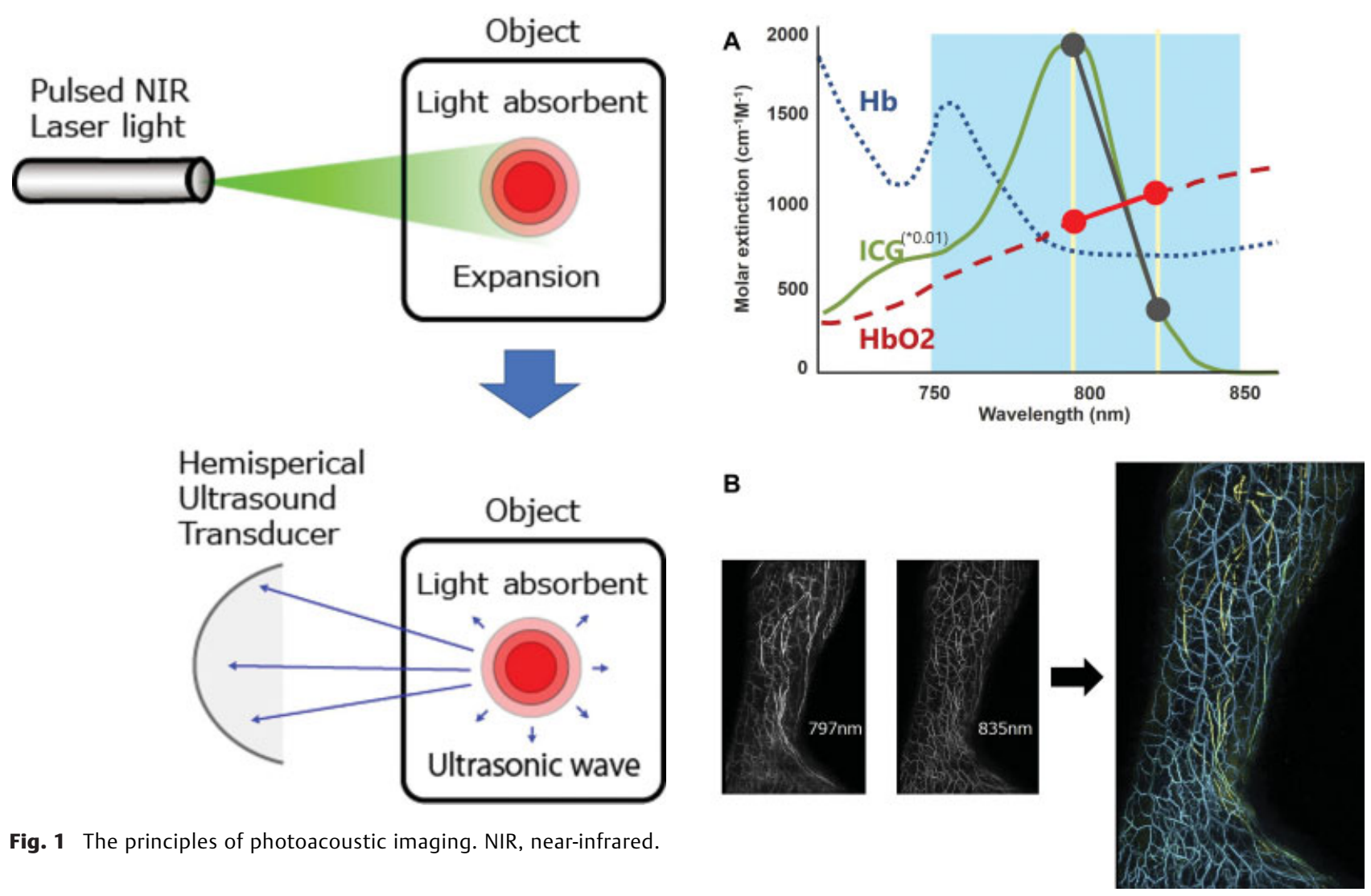

Fig. 1 The principles of photoacoustic imaging. NIR, near-infrared.

(5) This photoacoustic wave is detected by an ultrasound transducer outside the body to create an image. By receiving photoacoustic waves at multiple points during image construction, it is possible to noninvasively identify the location of light absorbers (hemoglobin and melanin, among others) in the body. ${ }^{1,2}$ The imaging test that uses PAI to depict lymphatic vessels is called photoacoustic lymphangiography (PAL). ${ }^{3-5}$

Lymphedema is a disease that causes edema in the limb due to congestion of lymphatic flow, and lymphaticovenular anastomosis (LVA) through the super microsurgical technique is one of the lymphedema treatments that allows stagnant lymphatic fluid to enter. There are various methods of LVA, and although there have been reports that LVA for improving edema can be performed in efferent lymphatic vessels by few anastomoses, especially in early-stage lymphedema, ${ }^{6,7}$ surgery with more anastomoses is generally more effective, and multiple LVA is generally recommended. ${ }^{8}$ However, to achieve multiple anastomoses, smooth anastomosis techniques and rapid identification of functional lymphatic vessels and veins are necessary. This rapid identification could be achieved by using PAL, which could identify lymphatic vessels and simultaneously depict veins.

Herein, we report findings on lymphatic vessels obtained using this new device, as well as their clinical application.

\section{Methods}

This prospective study was conducted from March 2018 at Keio University hospital. We recruited healthy volunteers

Fig. 2 Principle of color separation between lymphatic vessels and veins (Reprinted with permission from Suzuki Y, Kajita H, Konishi N et al. Subcutaneous lymphatic vessels in the lower extremities: comparison between photoacoustic lymphangiography and near-infrared fluorescence lymphangiography. Radiology 2020;295(2):469-474). (A) This graph shows relationship of wavelength and molar extinction of the object. Blood and lymph vessels are distinct by the differences in the molar extinction between indocyanine green (ICG) and hemoglobin ( $\mathrm{Hb})$. $\mathrm{HbO}_{2}$ : oxyhemoglobin. (B) Image of a 53-year-old female healthy volunteer. Left, lymphatic vessels are more prominently visualized at $797 \mathrm{~nm}$, allowing differentiation from blood vessels. Right, by comparing the difference of the figure, one can separate and colorize lymphatic and blood vessels. The yellow coloration signifies lymphatics, whereas the blue coloration signifies the vein.

and patients with lymphedema, and imaged the lymphatic vessels and veins of their lower extremities.

In this study, we used a PAI-05 device (currently undergoing product development). ${ }^{1}$

This system can irradiate lasers of two different wavelengths, making it possible to distinguish between blood and lymphatic vessels based on the difference in the intensity of the photoacoustic waves obtained from them at each wavelength. ${ }^{1,2,9}$ However, the transparency of the lymph fluid makes it impossible to delineate the lymph vessels. Therefore, as a light absorber, we used indocyanine green (ICG; Diagnogreen 0.5\%; Daiichi Pharmaceutical, Tokyo, Japan), which has also been used for NIRF lymphangiography. The absorbance of ICG increases at $797 \mathrm{~nm}$ and decreases at $835 \mathrm{~nm}$. By contrast, absorbance of hemoglobin does not significantly change between these two wavelengths (-Fig. 2A). At $797 \mathrm{~nm}$, the absorbance of ICG is significantly greater than that of 


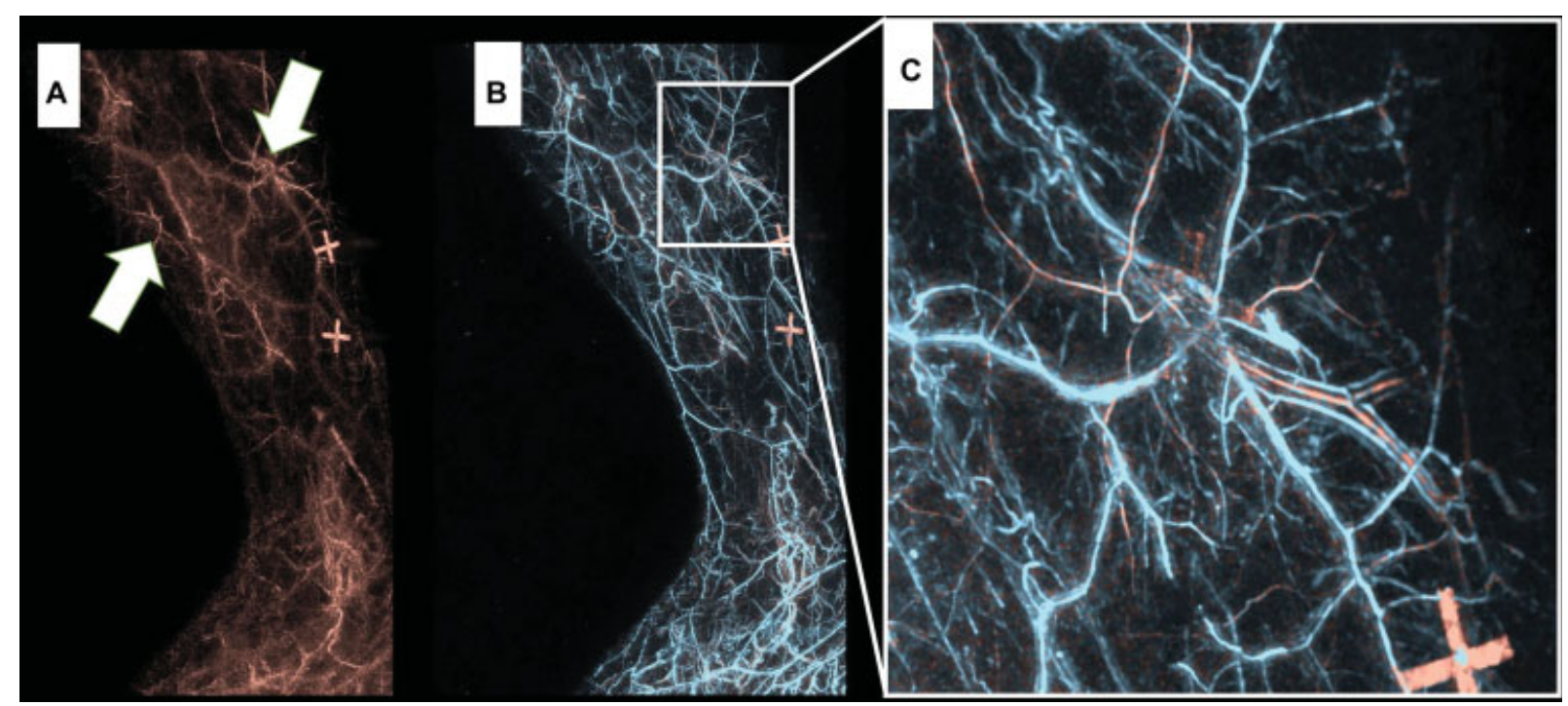

Fig. 3 Images of a 77-year-old male healthy volunteer: the red coloration signifies the artery, whereas the blue coloration signifies the vein. (A) Arterial imaging of the lateral side of the left extremity. The arrow points to the perforator artery. The vein signal remained slightly indistinct. (B) Photoacoustic image of the artery and vein. (C) Zoomed in image of the squared portion in - Fig. $\mathbf{3 B}$. One running artery and two running veins can be seen.

hemoglobin, allowing lymph vessels to be strongly depicted, whereas blood vessels are depicted more strongly at $835 \mathrm{~nm}$. By noting the difference between the images obtained from these two wavelengths, it became possible to distinguish between the lymph and blood vessels (-Fig. 2B). In addition, this imaging allows the threedimensional visualization of the relationship between the lymphatics and the veins (-Video 1 ).

\section{Video 1}

360-degree view of the - Fig. 2B. One can observe the three-dimensional relationship between lymphatics and veins. (Reproduced with permission from Suzuki Y, Kajita H, Konishi N et al. Subcutaneous lymphatic vessels in the lower extremities: comparison between photoacoustic lymphangiography and near-infrared fluorescence lymphangiography. Radiology 2020;295 (2):469-474). Online content including video sequences viewable at: https://www.thieme-connect.com/ products/ejournals/html/10.1055/s-0041-1736518.

Similarly, for distinguishing arteries from veins, the $756 \mathrm{~nm}$ and $797 \mathrm{~nm}$ wavelengths can be used, because of the difference in the absorbance of oxidized hemoglobin and reduced hemoglobin becomes greater at $756 \mathrm{~nm}$ wavelengths (-Fig. 3). ${ }^{1}$

These arterial and venous data are clinically useful, e.g., when dissecting the perforator flap. In particular, the suprafascial area can be identified; therefore, the vascular data are useful to make the flap thin. ${ }^{10}$

This study was conducted with the approval of the appropriate local ethics committee. Oral and written informed consent was obtained from all participants.

\section{Results}

\section{Imaging by Photoacoustic Lymphangiography}

The Lymphatics and Veins in Healthy Individuals The NIRF showed a linear pattern (-Fig. 4A), reflecting good functioning of the collecting lymphatic vessels. By contrast, the PAL more clearly showed the collecting lymphatics (-Fig. 4B) along with the veins (-Fig. 4C). ${ }^{3,11}$

The Lymphatics and Veins in Patients with Lymphedema The figures obtained from patients with lymphedema differed with respect to their lymphatic function. In particular, dermal backflow is a characteristic finding in lymphedema. In addition to NIRF, the dermal backflow can often be observed in PAL in the limbs with lymphedema (-Fig. 5A).

Moreover, our device was able to observe the object threedimensionally; hence, collective lymphatic vessels running under the dermal backflow were also identified. Furthermore, not only was PAL regurgitation continuity from collective lymphatic vessels to precollector lymphatic vessels demonstrated, but the relationship between these lymphatics and veins was also observed ( $\mathbf{- F i g . 5 B}$ and $\mathbf{C}$ ). In addition, PAL can digitally eliminate the dermal backflow from the image. Thus, hidden collective lymphatic vessels that run under dermal backflow could also be observed (- Fig. 5D).

However, in patients with advanced lymphedema, the function of the lymphatic vessels is deteriorated, and neither the lymphatic vessels nor the dermal backflow could be contrasted (-Fig. 6A-6C).

\section{Application of Photoacoustic Imaging}

\section{Preoperative Mapping}

PAL was performed before LVA surgery, and lymphatic vessels were well-visualized on the images ( - Fig. 7A and B). For 

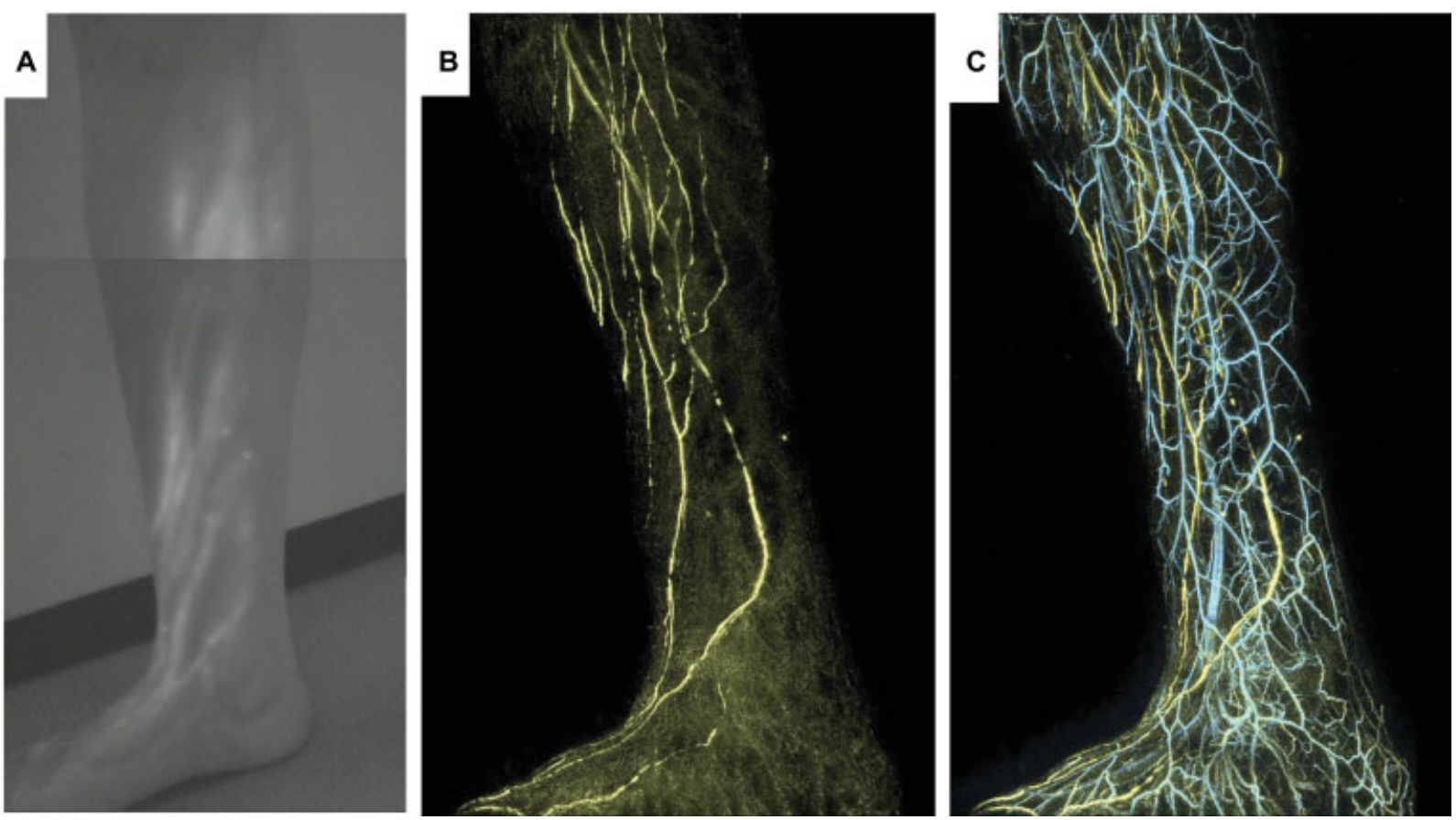

Fig. 4 Images of a 56-year-old female (Reprinted with permission from Suzuki Y, Kajita H, Oh A, et al. Use of photoacoustic imaging to determine the effects of aging on lower extremity lymphatic vessel function. J Vasc Surg Venous Lymphat Disord 2022;10(1):125-130). (A) Near-infrared fluorescent lymphangiography of the medial side of the right lower leg. (B) Photoacoustic lymphangiography of only the lymphatic vessels. (C) Photoacoustic lymphangiography of the lymphatics and veins.
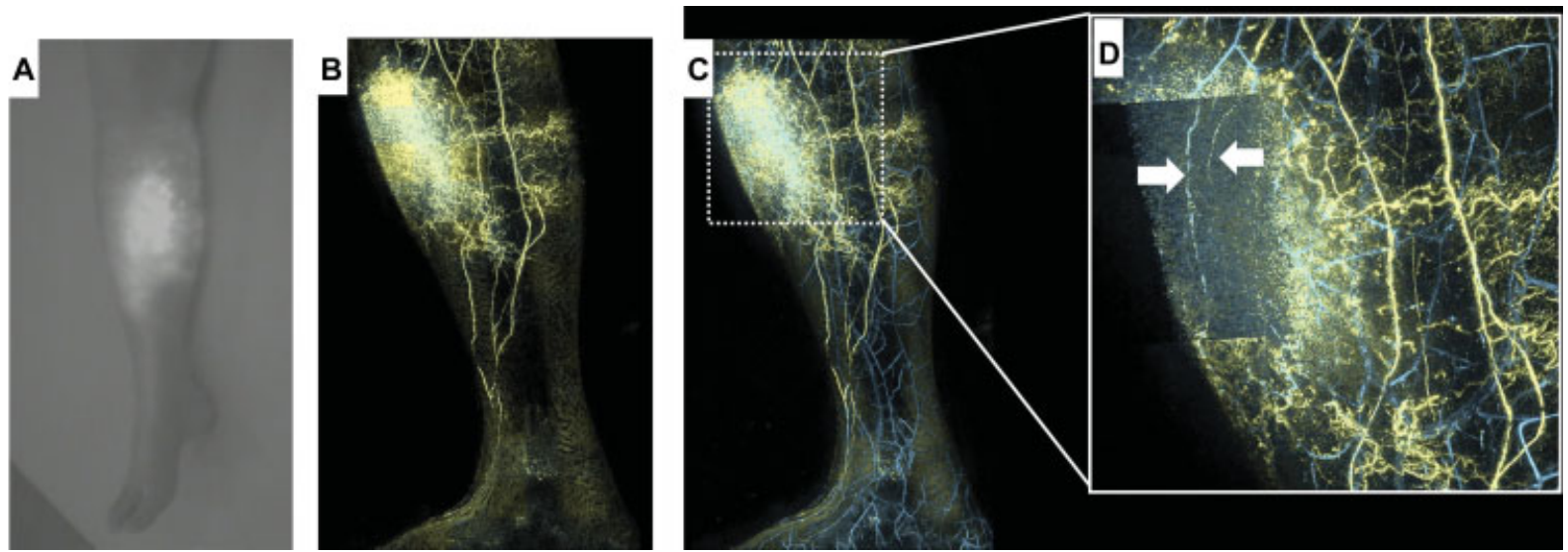

Fig. 5 Images of a 37-year-old female patient with lymphedema (Reprinted with permission from Suzuki Y, Kajita H, Konishi $\mathrm{N}$ et al. Subcutaneous lymphatic vessels in the lower extremities: comparison between photoacoustic lymphangiography and near-infrared fluorescence lymphangiography. Radiology 2020;295(2):469-474). (A) Near-infrared fluorescent lymphangiography imaging revealed dermal backflow in the lower extremity. (B) Photoacoustic lymphangiography demonstrated both a well-contrasted lymphatic vessels, as well as dermal backflow in the anterior side of the lower extremity. (C) Photoacoustic lymphangiography is able to show the relationship between the lymph vessels and the subcutaneous veins. (D) The figure taken by PAI-05 can digitally delete the dermal backflow. Thus, we can find the structures under dermal backflow. White arrow shows collective lymphatic vessel that was hidden by dermal backflow.

example, at this incision site, the subcutaneous vein and well-depicted lymphatic vessel were identified in the preoperative images. Moreover, the lymphatic vessels had been confirmed at the depth of this vein (-Fig. 7C, - Video 2) before surgery. Hence, we were able to identify the lymphatic vessels in the deeper side of this vein. The images obtained preoperatively and the actual intraoperative findings were well matched, and a good anastomosis was performed (-Fig. 7D-F).

\section{Video 2}

360-degree view of the -Fig. 7D. This three-dimensional data revealed the fact that the lymphatic vessel run deeper than veins became clear. Online content including video sequences viewable at: https://www. thieme-connect.com/products/ejournals/html/ 10.1055/s-0041-1736518. 

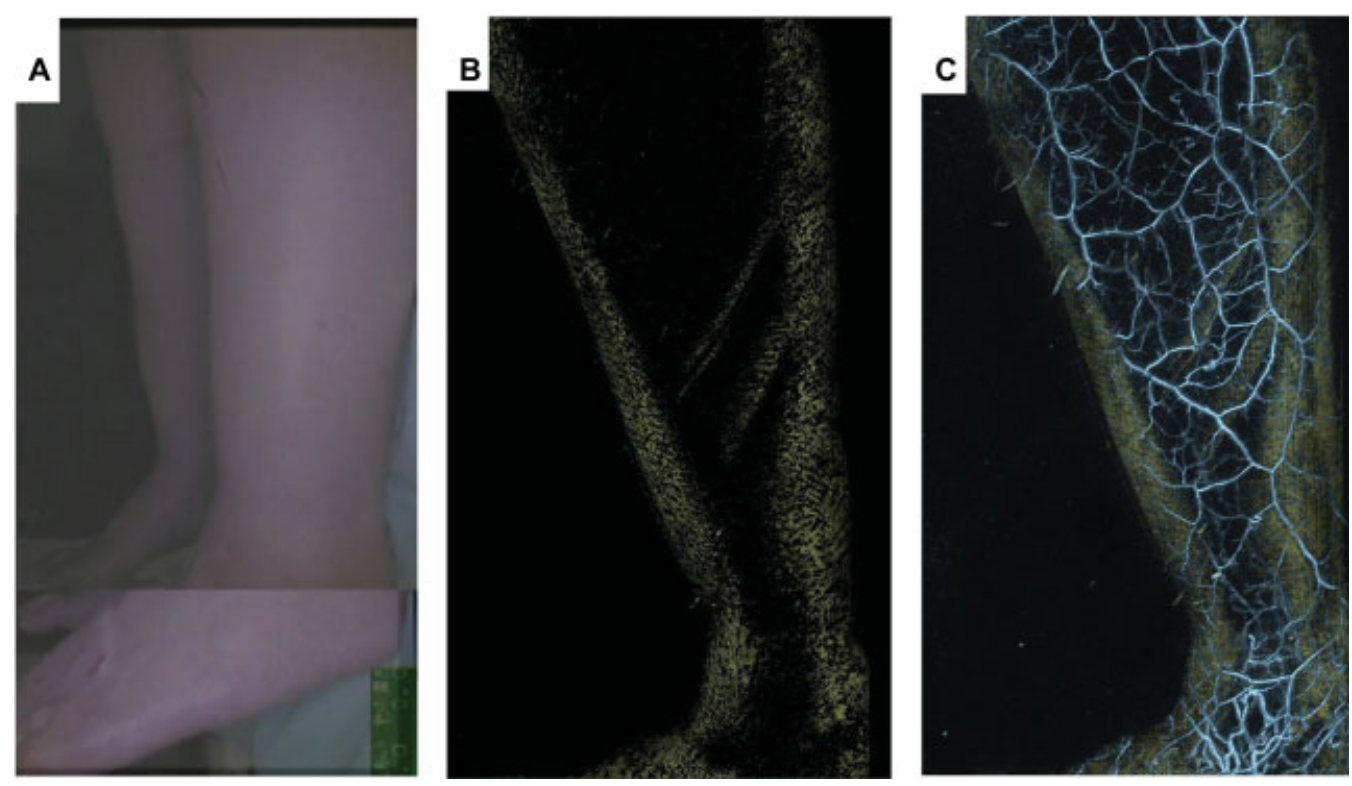

Fig. 6 Images of a 64-year-old female patient with lymphedema. (A) Near-infrared fluorescent lymphangiography imaging does not reveal any enhanced lymphatic vessel. (B) Photoacoustic lymphangiography of only the lymphatic vessels. However, no lymphatic vessels are identified. (C) Photoacoustic lymphangiography of the lymphatics and veins.
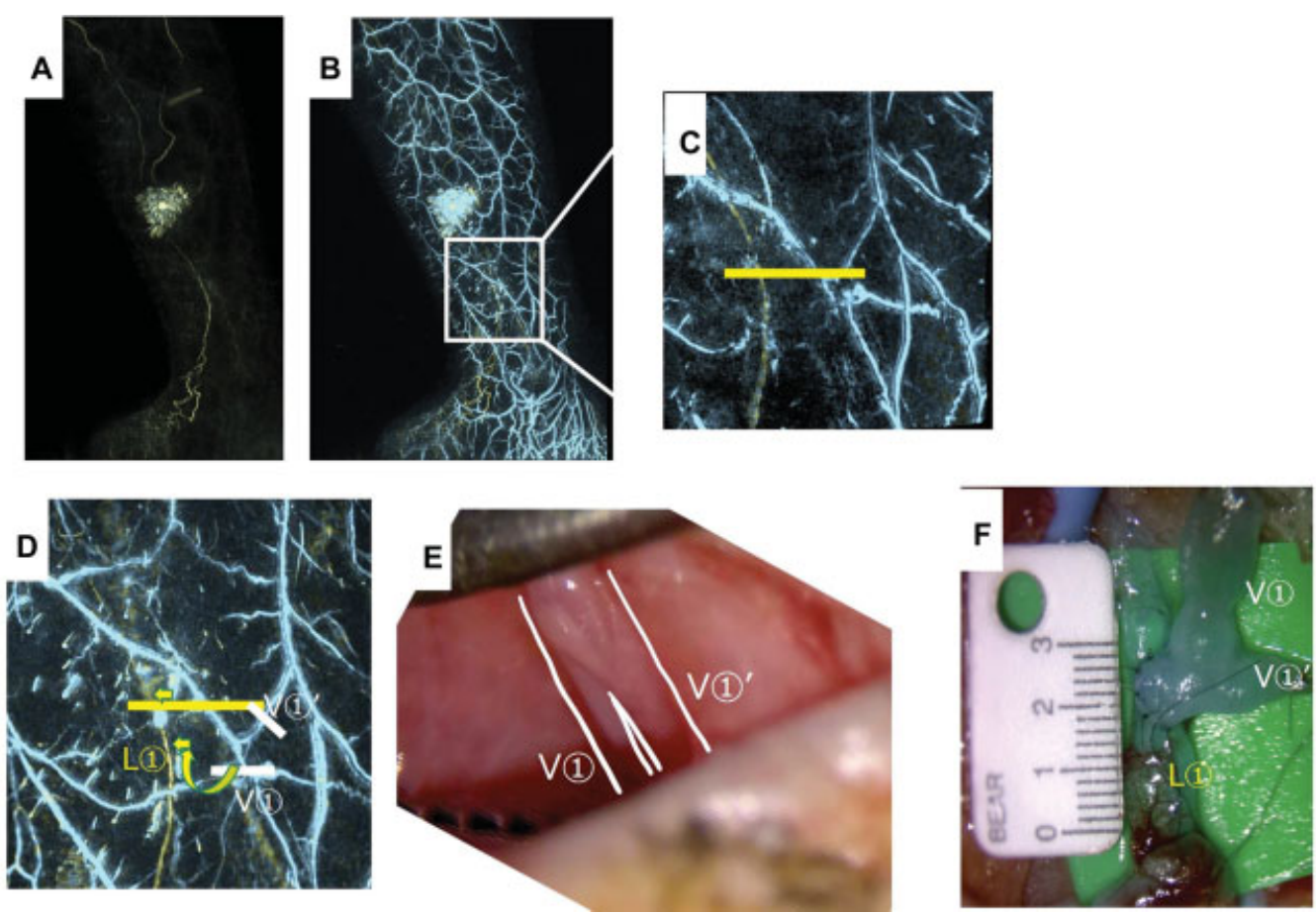

Fig. 7 Images of a 77-year-old female patient with secondary lymphedema due to cervical cancer. (A) Preoperative mapping by photoacoustic lymphangiography of only the lymphatic vessels. (B) Preoperative mapping by photoacoustic lymphangiography of the lymphatics and veins. (C) Magnified image of the anastomosis site. (D) Lymphatic vessel anastomosed later was named as L1 and vein as V1. The veins (V1 and V1') was transposed toward the lymph vessels following the round arrows. (E) Intraoperative finding. The same bifurcated vein depicted in - Fig. 7D was identified. (F) Referring to preoperative picture, identification of the lymphatic vessel and venule was easily performed. Thus, good lymphaticovenular anastomosis was achieved.

\section{Postoperative Evaluation of LVA}

When the anastomosis was observed with PAL, the findings demonstrated the contact between the lymphatic fluid (ICG) and the blood at the border of the anastomosis. ${ }^{12}$ When the anastomosis is obstructed, hemoglobin and ICG signals will not be detected at the border, which is thought to cause a gap between the lymph fluid and blood at the anastomosis. In our case, the lymph fluid and blood were joined, suggesting that the anastomosis was patent ( $\mathbf{- F i g . ~} \mathbf{8 A - C}$ ).

\section{Discussion}

Lymphatic vessels have historically been studied in detail by anatomical studies of lymphatic vessels in cadavers, using 



Fig. 8 Photoacoustic lymphangiography after 1 month of lymphaticovenular anastomosis surgery. (Reprinted with permission from Suzuki Y, Kajita $\mathrm{H}$, Kono $\mathrm{H}$, et al. The direct observation of lymphaticovenular anastomosis patency with photoacoustic lymphangiography. Plast Reconstr Surg Glob Open 2021;9(1):e3348). (A) Anastomosis site was showed in white square. (B) Intraoperative findings. Lymphaticovenular end-to-end anastomosis had performed. (C) Magnified and rotated image of the anastomosis site. In, Fig. B, the skin around anastomosis site was pulled to get better view for the anastomosis, and the tension was removed since wound closure. Hence, the anastomosis may be depicted as sagging. The lymphatic vessels in yellow gradually cross the anastomosis and connect to the veins.

mercury, or more recently, lead oxide. ${ }^{13}$ However, in living humans, observing lymphatics is extremely difficult because the lymph vessels are tiny and the lymph fluid flowing inside the vessels is transparent. The PAI-05 implemented in this study uses ICG as a light absorber, allowing a clearer evaluation of subcutaneous lymphatic vessels compared with existing modalities.

Our group was the first in the world to successfully capture clear three-dimensional images of a wide range of human lymphatic vessels using PAI. ${ }^{5}$ This device has a high resolution $(0.2 \mathrm{~mm})$ and is capable of individually identifying multiple lymphatic vessels. Handheld PAI devices have also been applied to lymphedema treatment, but the area that can be imaged was only up to $15 \mathrm{~mm}$ in diameter. $^{14}$

Previously, imaging modalities such as lymphoscintigraphy, ${ }^{15,16}$ NIRF lymphangiography, ${ }^{17-19}$ magnetic resonance lymphangiography (MRL), ${ }^{16,20-22}$ and ultrasonography ${ }^{23,24}$ have been used to observe lymphatic vessels for lymphedema treatment (- Table 1). However, although lymphoscintigraphy can evaluate the entire lower extremity, the details of each lymphatic vessel remain unknown. NIRF lymphangiography has the advantage of evaluating lymphatic vessels in real time and is probably the most commonly used technique for preoperative LVA mapping. However, an NIRF cannot provide information on the depth of the lymphatics. MRL 
Table 1 Comparison of imaging modalities for lymphatic vessels

\begin{tabular}{|c|c|c|}
\hline & Pros & Cons \\
\hline LS & Enables complete evaluation of the lower extremities & Difficult to identify details of individual lymphatic vessels \\
\hline NIRF & $\begin{array}{l}\text { Real time imaging } \\
\text { Widely used in lymphatic surgery }\end{array}$ & Provides only two-dimensional information \\
\hline MRL & $\begin{array}{l}\text { Detailed resolution and wide imaging range in three } \\
\text { dimensions }\end{array}$ & $\begin{array}{l}\text { Smaller lymphatic vessels are not evident at early lymph- } \\
\text { edema stages } \\
\text { Venous contamination may lead to misidentification of } \\
\text { lymphatics }\end{array}$ \\
\hline US & $\begin{array}{l}\text { Simultaneous identification of lymphatics and veins } \\
\text { No contrast agent required }\end{array}$ & $\begin{array}{l}\text { Reproducibility issues because accuracy of image rendi- } \\
\text { tion depends on the skill of practitioner }\end{array}$ \\
\hline PAL & $\begin{array}{l}\text { High-definition images with a resolution of } 0.2 \mathrm{~mm} \text { enable } \\
\text { simultaneous identification of three-dimensional lym- } \\
\text { phatics and veins } \\
\text { Video recording is possible }\end{array}$ & $\begin{array}{l}\text { Imaging range is limited } \\
\text { The device is still in its development stage }\end{array}$ \\
\hline
\end{tabular}

Abbreviations: LS, lymphoscintigraphy; MRL, magnetic resonance lymphangiography; NIRF, near-infrared fluorescence lymphangiography; PAL, photoacoustic lymphangiography; US, ultrasonography.

is expected to develop in the future because of its detailed resolution and wide imaging range in three dimensions, but at present, it has difficulties visualizing small lymphatic vessels; moreover, due to venous contamination, some veins are unavoidably enhanced, which may lead to misidentification of lymphatic vessels. Ultrasonography has been applied to the preoperative evaluation of LVA and is able to simultaneously identify lymphatics and veins, while the other three modalities cannot detect veins in detail. Lymphatic structures can also be visualized if ultra-high-frequency ultrasonography is used. However, lymphatic vessels that can be identified vary depending on the skill of the practitioner, and there is a reproducibility problem.

Compared with these modalities, PAL is useful in LVA, considering its detailed imaging ability to identify lymphatic vessels and to simultaneously depict veins. ${ }^{25}$ In preoperative PAL, it is possible to simultaneously visualize the lymphatic vessels and veins to be anastomosed. Moreover, the threedimensional positional relationship of both lymphatic vessels and veins can be clearly seen, which allowed quick identification of each, leading to efficient LVA. Patients with lymphedema have expanded limb appearance, risk of infection (e.g., cellulitis), pain, and lymphorrhea. The main goal of lymphedema surgery is to relieve the patients' symptoms and decrease limb circumference of the extremity. To achieve this result, attaining a patent anastomosis should be the premise of the surgery. If all anastomoses were obstructed, the patient's edema would not improve. Hence, the patency of the anastomosis needs to be assessed. Nevertheless, despite its importance, assessing anastomotic patency is difficult.

Although existing reports assessing anastomotic patency by NIRF have reported patency rates of 30 to $50 \%$, the accuracy of patency rate for all anastomoses remains unknown because it is difficult to assess when the anastomosis site is hidden by dermal backflow. ${ }^{26-28}$

PAL can digitally delete dermal backflow and be less affected by its influence; therefore, PAL could be used to better judge lymphatic anastomosis patency than NIRF. ${ }^{12}$ Both techniques still require ICG for detecting lymphatic vessels; hence, we cannot judge the patency in nonenhanced lymphatic vessels.

PAL is still a developing technology, and at present, the imaging range is too limited for preoperative mapping using PAL alone, and it is still difficult to precisely adjust the PAL figures and the patient's limb for the planning of the LVA surgery. However, the clear image provided by this modality can be useful for lymphatic surgeons, and further development is expected in the future.

\section{Conclusion}

In this article, we reported the use of PAI to delineate lymphatic vessels and their clinical application. This technique can visualize lymphatic vessels with higher resolution than conventional imaging techniques and in three dimensions. Further development of this technique is expected to play a major role in the treatment of lymphedema.

\section{Funding}

This research was funded by a grant from ImPACT Program of Council for Science, Technology and Innovation (Cabinet Office, Government of Japan) and the Japan Agency for Medical Research and Development (AMED) JP21 he2302002.

\section{Conflict of Interest}

PAI-05 that we used in this manuscript was provided free of charge from Luxonus Inc. to Keio University School of Medicine. Dr. Aiso is CEO of the Luxonus Inc. Dr. Suzuki reports grants from ImPACT Program of Council for Science, Technology and Innovation (Cabinet Office, Government of Japan), grants from Grant from AMED, during the conduct of the study. Dr. Kajita reports grants from ImPACT Program of Council for Science, Technology and Innovation (Cabinet Office, Government of Japan), grants from Tobikai Medical 
Corporation, grants from Japan Agency for Medical Research and Development (AMED), non-financial support from Luxonus, Inc, during the conduct of the study. In addition, Dr. Kajita has a patent PCT/JP2019/032560 pending, a patent PCT/JP2019/032564 pending, a patent PCT/ JP2019/032586 pending, a patent PCT/JP2019/013968 pending, a patent PCT/JP2019/013953 pending, a patent PCT/JP2019/013940 pending, a patent JP2018-157800 pending, a patent U.S. Patent Application No. 16/542,681 pending, a patent JP2018-157798 pending, a patent JP2018-157797 pending, a patent JP2018-157794 pending, a patent JP2018-157785 pending, a patent JP2018-157755 pending, a patent JP2018-157752 pending, a patent JP2018-155043 pending, a patent JP2018-155034 pending, and a patent JP2018-155033 pending. Dr. Imanishi reports grants from ImPACT Program of Council for Science, Technology and Innovation (Cabinet Office, Government of Japan), grants from Tobikai Medical Corporation, grants from Japan Agency for Medical Research and Development (AMED), non-financial support from Luxonus, Inc, during the conduct of the study. In addition, Dr. Imanishi has a patent PCT/JP2019/032560 pending, a patent PCT/JP2019/ 032564 pending, a patent PCT/JP2019/032586 pending, a patent PCT/JP2019/013968 pending, a patent PCT/JP2019/ 013953 pending, a patent PCT/JP2019/013940 pending, a patent JP2018-157800 pending, a patent U.S. Patent Application No. 16/542,681 pending, a patent JP2018-157798 pending, a patent JP2018-157797 pending, a patent JP2018-157794 pending, a patent JP2018-157785 pending, a patent JP2018-157755 pending, a patent JP2018-157752 pending, a patent JP2018-155043 pending, a patent JP2018-155034 pending, and a patent JP2018-155033 pending. Dr. Sakuma reports grants from Japan Agency for Medical Research and Development (AMED), grants from ImPACT Program of Council for Science, Technology and Innovation (Cabinet Office, Government of Japan), nonfinancial support from Luxonus, Inc, non-financial support from Canon, Inc, outside the submitted work. Dr. Kishi reports grants from Japan Agency for Medical Research and Development (AMED), grants from ImPACT Program of Council for Science, Technology and Innovation (Cabinet Office, Government of Japan), non-financial support from Luxonus, Inc, non-financial support from Canon, Inc, during the conduct of the study. Dr. Okabe reports grants from Japan Agency for Medical Research and Development (AMED), grants from ImPACT Program of Council for Science, Technology and Innovation (Cabinet Office, Government of Japan), non-financial support from Luxonus, Inc, non-financial support from Canon, Inc, during the conduct of the study. Dr. Aiso reports grants from Japan Agency for Medical Research and Development (AMED), grants from ImPACT Program of Council for Science, Technology and Innovation (Cabinet Office, Government of Japan), CEO and shareholder of Luxonus, Inc, non-financial support from Canon, Inc, during the conduct of the study; personal fees and other from Luxonus, Inc., outside the submitted work; and is getting personal fee from the company, as well as is a shareholder of the company.

\section{References}

1 Nagae K, Asao Y, Sudo Y, et al. Real-time 3D photoacoustic visualization system with a wide field of view for imaging human limbs. F1000 Res 2018;7:1813

2 Zackrisson S, van de Ven SMWY, Gambhir SS. Light in and sound out: emerging translational strategies for photoacoustic imaging. Cancer Res 2014;74(04):979-1004

3 Suzuki Y, Kajita H, Konishi N, et al. Subcutaneous lymphatic vessels in the lower extremities: comparison between photoacoustic lymphangiography and near-infrared fluorescence lymphangiography. Radiology 2020;295(02):469-474

4 Kajita H, Oh A, Urano M, et al. Photoacoustic lymphangiography. J Surg Oncol 2020;121(01):48-50

5 Kajita H, Kishi K. High-resolution imaging of lymphatic vessels with photoacoustic lymphangiography. Radiology 2019;292(01):35

6 Akita S, Yamaji Y, Kuriyama M, et al. Intraoperative detection of efferent lymphatic vessels emerging from lymph node during lymphatic microsurgery. J Reconstr Microsurg 2019;35(05): 372-378

7 Yamamoto T, Yamamoto N, Yamashita M, Furuya M, Hayashi A, Koshima I. Efferent lymphatic vessel anastomosis: supermicrosurgical efferent lymphatic vessel-to-venous anastomosis for the prophylactic treatment of subclinical lymphedema. Ann Plast Surg 2016;76(04):424-427

8 Mihara M, Hara H, Tange S, et al. Multisite lymphaticovenular bypass using supermicrosurgery technique for lymphedema management in lower lymphedema Cases. Plast Reconstr Surg 2016;138(01):262-272

9 Tsuge I, Saito S, Sekiguchi H, et al. Photoacoustic tomography shows the branching pattern of anterolateral thigh perforators in vivo. Plast Reconstr Surg 2018;141(05):1288-1292

10 Tsuge I, Saito S, Yamamoto G, et al. Preoperative vascular mapping for anterolateral thigh flap surgeries: a clinical trial of photoacoustic tomography imaging. Microsurgery 2020;40(03):324-330

11 Suzuki Y, Kajita H, Oh A, et al. Use of photoacoustic imaging to determine the effects of aging on lower extremity lymphatic vessel function. J Vasc Surg Venous Lymphat Disord 2022;10 (01):125-130

12 Suzuki Y, Kajita $H$, Kono $H$, et al. The direct observation of lymphaticovenular anastomosis patency with photoacoustic lymphangiography. Plast Reconstr Surg Glob Open 2021;9(01): e3348

13 Suami H, Taylor GI, Pan WR. A new radiographic cadaver injection technique for investigating the lymphatic system. Plast Reconstr Surg 2005;115(07):2007-2013

14 Giacalone G, Yamamoto T, Belva F, Hayashi A. Bedside 3D visualization of lymphatic vessels with a handheld multispectral optoacoustic tomography device. J Clin Med 2020;9(03):815

15 Maegawa J, Mikami T, Yamamoto Y, Satake T, Kobayashi S. Types of lymphoscintigraphy and indications for lymphaticovenous anastomosis. Microsurgery 2010;30(06):437-442

16 Notohamiprodjo M, Weiss M, Baumeister RG, et al. MR lymphangiography at $3.0 \mathrm{~T}$ : correlation with lymphoscintigraphy. Radiology 2012;264(01):78-87

17 Yamamoto T, Narushima M, Doi K, et al. Characteristic indocyanine green lymphography findings in lower extremity lymphedema: the generation of a novel lymphedema severity staging system using dermal backflow patterns. Plast Reconstr Surg 2011;127(05):1979-1986

18 Hara H, Mihara M, Seki Y, Todokoro T, Iida T, Koshima I. Comparison of indocyanine green lymphographic findings with the conditions of collecting lymphatic vessels of limbs in patients with lymphedema. Plast Reconstr Surg 2013;132(06): 1612-1618

19 Narushima M, Yamamoto T, Ogata F, Yoshimatsu H, Mihara M, Koshima I. Indocyanine green lymphography findings in limb lymphedema. J Reconstr Microsurg 2016;32(01):72-79 
20 Ruehm SG, Schroeder T, Debatin JF, Interstitial MR. Interstitial MR lymphography with gadoterate meglumine: initial experience in humans. Radiology 2001;220(03):816-821

21 Neligan PC, Kung TA, Maki JH. MR lymphangiography in the treatment of lymphedema. J Surg Oncol 2017;115(01):18-22

22 Yasunaga Y, Nakajima Y, Mimura S, Yuzuriha S, Kondoh S. Magnetic resonance lymphography as three-dimensional navigation for lymphaticovenular anastomosis in patients with leg lymphedema. J Plast Reconstr Aesthet Surg 2021;74(06):1253-1260

23 Mihara M, Hara H, Kawakami Y. Ultrasonography for classifying lymphatic sclerosis types and deciding optimal sites for lymphatic-venous anastomosis in patients with lymphoedema. J Plast Reconstr Aesthet Surg 2018;71(09):1274-1281

24 Hayashi A, Hayashi N, Yoshimatsu H, Yamamoto T. Effective and efficient lymphaticovenular anastomosis using preoperative ultrasound detection technique of lymphatic vessels in lower extremity lymphedema. J Surg Oncol 2018;117(02): 290-298

25 Oh A, Kajita H, Matoba E, et al. Photoacoustic lymphangiography before and after lymphaticovenular anastomosis. Arch Plast Surg 2021;48(03):323-328

26 Maegawa J, Yabuki Y, Tomoeda H, Hosono M, Yasumura K. Outcomes of lymphaticovenous side-to-end anastomosis in peripheral lymphedema. J Vasc Surg 2012;55(03):753-760

27 Suzuki Y, Sakuma H, Yamazaki S. Evaluation of patency rates of different lymphaticovenous anastomosis techniques and risk factors for obstruction in secondary upper extremity lymphedema. J Vasc Surg Venous Lymphat Disord 2019;7(01):113-117

28 Suzuki Y, Sakuma H, Yamazaki S. Comparison of patency rates of lymphaticovenous anastomoses at different sites for lower extremity lymphedema. J Vasc Surg Venous Lymphat Disord 2019;7 (02):222-227 